\title{
POINCARÉ AND ALGEBRAIC GEOMETRY
}

\author{
BY PHILLIP A. GRIFFITHS ${ }^{1}$
}

\begin{abstract}
ABSTRaCr. A few of the contributions of Poincare to algebraic geometry are described, with emphasis on his late work on normal functions. A very brief description of some recent works is also given.
\end{abstract}

Although the subject of algebraic geometry was not one of Poincarés major preoccupations, his work in the field showed characteristic insight and brilliance and certainly has had a lasting effect. I shall briefly describe his major contributions, with special emphasis on the two papers on normal functions which constituted Poincaré's last published mathematical work.

In general I have tried to follow standard current notations. Unless mentioned to the contrary, homology and cohomology are with $\mathbf{Q}$ coefficients.

At the end there is a list of some of Poincaré's major papers in algebraic geometry together with the other bibliographical items referred to in the text.

As will be clear from the discussion below, the decision to emphasize Poincaré's work on normal functions reflects my own particular interest.

(a) Let me begin by recalling the period during which Poincaré worked. This was a particularly active time in algebraic geometry, especially for the study of algebraic surfaces. In the preceding thirty years, beginning with Riemann's thesis the theory of algebraic functions of one variable, or as we now know it, algebraic curves, had been developed by Riemann, Max Noether, and many others to the point where-aside from moduli questions - the theory had assumed much the form that we find it today. Beginning in the 1890's Poincaré's colleague, E. Picard, had begun his monumental work on transcendental algebraic geometry in higher dimensions, especially on surfaces, that among other things led to the Picard-Fuchs equation and Picard-Lefschetz transformation, the algebraic de Rham theorem for smoothly compactifiable affine varieties and subsequent theory of single and double integrals of the second kind on surfaces, and the Picard variety and Picard number. Meanwhile, in Italy the birational theory of algebraic surfaces was well underway in the work of Castelnuovo, Enriques, Severi (at a slightly later time), and the other members of the Italian school.

It is clear that Poincare was well abreast of these developments, and it seems to me that his own work in algebraic geometry was frequently in

Presented to the Symposium on the Mathematical Heritage of Henri Poincaré, April 7-10, 1980; received by the editors November 1, 1980.

1980 Mathematics Subject Classification. Primary

${ }^{1}$ Research partially supported by NSF Grant MCS7707782. 
response to this activity. For example, as will be explained below, his idea of normal functions was devised to give the first complete proof of one of the main results of Picard and Castelnuovo-Enriques, the theorem that the Picard variety of a surface has dimension equal to the irregularity.

(b) Poincaré wrote a number of papers, several in collaboration with Picard, concerning abelian varieties and Jacobians of curves. As was characteristic of much of his work, several of these papers involved interaction between various branches of mathematics. For example, his studies on abelian functions led to the Cousin problems in several complex variables, and his work on translation-type surfaces in abelian varieties was instrumental in the theory of webs about which Chern will speak.

In algebraic geometry proper, at least two results from the area of abelian varieties and Jacobians are noteworthy. One is

(1) Poincaré's complete reducibility theorem [5]: Given an abelian subvariety $A^{\prime}$ of an abelian variety $A$, there exists a finite covering

$$
\tilde{A} \stackrel{\pi}{\rightarrow} A
$$

and abelian subvariety $A^{\prime \prime} \subset \tilde{A}$ such that

$$
\tilde{A} \cong \pi^{-1}\left(A^{\prime}\right) \times A^{\prime \prime} .
$$

This is a clear forerunner of the modern version that states: the category of polarized Hodge structures $/ \mathbf{Q}$ is semisimple.

A second result concerns the Jacobian variety $J$ of a smooth algebraic curve $C$ of genus $g$. Recall that there is a holomorphic mapping $u: C \rightarrow J$ defined by

$$
u(p)=\left(\int_{p_{0}}^{p} \omega_{1}, \ldots, \int_{p_{0}}^{p} \omega_{g}\right)
$$

where $p_{0} \in C$ is a base point and $\omega_{1}, \ldots, \omega_{g}$ is a basis for the holomorphic differentials on $C$. If $C_{d}$ denotes the set of all unordered $d$-tuples of points

$$
D=p_{1}+\cdots+p_{d} \text {, }
$$

it may be shown that $C_{d}$ has a natural complex manifold structure to which the mapping (2) may be extended by the formation of abelian sums. Thus

$$
u: C_{d} \rightarrow J
$$

is defined by

$$
u\left(p_{1}+\cdots+p_{d}\right)=\left(\sum_{i} \int_{p_{0}}^{p_{i}} \omega_{1}, \ldots, \sum_{i} \int_{p_{0}}^{p_{i}} \omega_{g}\right) .
$$

Definition. For $1 \leqslant d \leqslant g$ we define the subvariety $W_{d} \subset J$ to be the image variety of the proper holomorphic mapping (3).

Equivalently, $W_{d}$ is the translation-type subvariety of $J$ given by sums of $d$ points on the curve $u(C)$; i.e.

$$
W_{d}=\left\{u\left(p_{1}\right)+\cdots+\left(p_{d}\right): p_{i} \in C\right\} .
$$

The Jacobi inversion theorem states that

$$
W_{g}=J \text {. }
$$

More precisely, for $d=g$ the mapping (3) is birational. 
Next, if $\Theta \subset J$ is the divisor of zeroes of Riemann's theta function, then Riemann's theorem is

$$
W_{g-1}=\Theta+\kappa,
$$

where the right-hand side of (5) is translation of $\Theta$ by the vector $\kappa$ of Riemann constants.

Poincaré gave the following extension of (4) and (5) to all $d \leqslant g$ :

(6) Poincaré's formula [6]. If $\theta \in H^{2}(J)$ and $w_{d} \in H^{2(g-d)}(J)$ are the fundamental cohomology classes of $\Theta$ and $W_{d}$ respectively, then

$$
w_{d}=\theta^{g-d} /(g-d) !
$$

When $d=g$ this implies that

$$
u: C_{g} \rightarrow J
$$

has degree one, and when $d=g-1$ (7) gives that

$$
w_{g-1}=\theta \text {. }
$$

Riemann's theorem (5) is then a consequence of the fact that the Jacobian of a curve is a principally polarized abelian variety.

It is perhaps worth pointing out that (6) could not have even been properly formulated before Poincaré's introduction of homology. This is also true of the next topic dealing with residues of double integrals.

In a similar vein we also note that Picard's great work [3] on algebraic functions of two variables would not have been possible had not Poincaré concurrently developed the necessary topological concepts.

(c) The next of Poincaré's investigations in algebraic geometry that I would like to discuss centers around what is now called the Poincaré residue operator. It is an elementary fact that, on the complex projective line $\mathbf{P}^{1}$ with linear coordinate $z$, the cohomology of the punctured sphere

$$
\mathbf{P}^{1}-\left\{a_{1}, \ldots, a_{N}\right\},
$$

$a_{\nu}$ distinct, is described by rational differential forms having poles at the $a_{\nu}$. More precisely, if we set

$$
p(z)=\prod_{\nu=1}^{N}\left(z-a_{\nu}\right)
$$

then every cohomology class is represented by a unique form

$$
w=q(z) d z / p(z), \quad \operatorname{deg} q(z) \leqslant N-2,
$$

that has simple poles at the $a_{\nu}$.

Motivated in part by his work on topology and in part by the aforementioned project of Picard concerning periods of rational integrals on algebraic surfaces, Poincaré [7] considered rational forms

$$
\omega=\frac{q(x, y) d x \wedge d y}{p(x, y)}, \quad \operatorname{deg} q(x, y) \leqslant N-3,
$$

on the projective plane $\mathbf{P}^{2}$. Here, $(x, y)$ are affine coordinates, $p(x, y)$ is an irreducible polynomial of degree $N$ defining an algebraic curve $D$, and the degree restriction in (8) is equivalent to saying that $\omega$ does not have a pole on 
the line at infinity. Poincare showed that the periods

$$
\iint_{\Gamma} \omega, \quad \Gamma \in H_{2}\left(\mathbf{P}^{2}-D\right)
$$

are all of the form

$$
\int_{\gamma} \operatorname{Res} \omega, \quad \gamma \in H_{1}(D)
$$

where

$$
\operatorname{Res} \omega=q d x /(\partial p / \partial y)=-q d y /(\partial p / \partial x) \text { on } p(x, y)=0
$$

defines a differential called the Poincaré residue. Here $\gamma$ is a 1-cycle in $D^{*}=D \backslash$ singular locus $\}$, and the cycles $\Gamma$ and $\gamma$ are related by $\Gamma=\tau(\gamma)$ where

$$
\tau: H_{1}\left(D^{*}\right) \rightarrow H_{2}\left(\mathbf{P}^{2}-D\right)
$$

is the mapping that assigns to $\gamma$ the boundary of that part of the solid $\varepsilon$-tube around $D^{*}$ that lies over $\gamma$.

Nowadays one considers the Poincaré residue sheaf sequence

$$
0 \rightarrow \Omega_{X}^{n} \rightarrow \Omega_{X}^{n}(D) \stackrel{\text { Res }}{\rightarrow} \Omega_{D}^{n-1} \rightarrow 0
$$

where $X$ is a smooth $n$-dimensional algebraic variety and $D \subset X$ is a divisor that, for simplicity of explanation, we assume to be smooth. Poincaré's case corresponds to $X=\mathbf{P}^{2}$, and the mapping (10)

$$
\omega \rightarrow \operatorname{Res} \omega
$$

is

$$
\text { Res: } H^{0}\left(\Omega_{X}^{n}(D)\right) \rightarrow H^{0}\left(\Omega_{D}^{n-1}\right) \text {. }
$$

His results concerning the periods of double integrals (9) are encoded in the long exact cohomology sequence of (12), together with Poincaré-Lefschetz duality and Hodge theory.

It is interesting to note that in his paper [7] on residues Poincaré raised the issue concerning periods

$$
\iint_{\Gamma} \frac{q(x, y) d x \wedge d y}{p(x, y)^{k+1}}, \quad \operatorname{deg} q(x, y) \leqslant(k+1) N-3,
$$

whose integrand has a higher order pole along $C$. Contrary to the one variable case, by subtracting exact forms it is not always possible to reduce the order of pole of $\omega$ to one, and Poincare mused on but did not entirely come to grips with this fact. It is now easy to explain the reason why: For a smooth hypersurface $D \subset \mathbf{P}^{n}$ of degree $N$ given in affine coordinates by

$$
p\left(x_{1}, \ldots, x_{n}\right)=0,
$$

we consider the mapping on cohomology

$$
\text { Res: } H^{n}\left(\mathbf{P}^{n}-D\right) \rightarrow H^{n-1}(D)
$$

dual to the "tube over cycle mapping"

$$
\tau: H_{n-1}(D) \rightarrow H_{n}\left(\mathbf{P}^{n}-D\right)
$$


encountered in the case $n=2$ in (11). The rational $n$-forms

$$
\Omega=\frac{q(x) d x_{1} \wedge \cdots \wedge d x_{n}}{p(x)^{k+1}}, \quad \operatorname{deg} q(x) \leqslant(k+1) n-(n+1),
$$

have poles of order $k+1$ along $D$, and they generate a vector subspace

$$
F^{n-k} H^{n}\left(\mathbf{P}^{n}-D\right) \subset H^{n}\left(\mathbf{P}^{n}-D\right) \text {. }
$$

It can be proved (cf. [8]) that the images

$$
\operatorname{Res}\left(F^{n-k} H^{n}\left(\mathbf{P}^{n}-D\right)\right)=F^{(n-1-k)} H_{\text {prim }}^{n-1}(D)
$$

define the Hodge filtration on the primitive cohomology of $D$.

This result, which generalizes to any sufficiently ample smooth divisor $D$ in a smooth variety $X$, provides a useful link between the transcendental Hodge theory of $D$ and the algebro-geometric notion of linear systems on $X$.

(d) We now turn to Poincaré's magnificent works [9 and 10] on normal functions. As mentioned above, these papers appeared just before his premature death while still at the height of his mathematical powers. Upon reading the last section of the second paper it is clear, at least with the benefit of hindsight, that he was "within $\varepsilon$ " of the Lefschetz $(1,1)$ theorem, and indeed, as we shall see, his normal functions provided the tool for the proof of this famous result.

We consider an algebraic family $\left\{C_{t}\right\}_{t \in T}$ of algebraic curves of which a general member is smooth. In practice, $T$ will usually also be a curve, and we shall denote by $t_{1}, \ldots, t_{N}$ the critical points for which the curves $C_{t_{1}}, \ldots, C_{t_{N}}$ are singular. For an important concrete example, we consider a smooth algebraic surface $S \subset \mathbf{P}^{n}$ and take $\left\{C_{t}\right\}_{t \in T}$ to be a general pencil of hyperplane sections. In coordinates one may think of the projection of $S$ in a general $\mathbf{P}^{3}$ to be given by

$$
p(x, y, z)=0
$$

in such a way that the curve $C_{t}$ is the section by the plane

$$
z=t \text {. }
$$

Associated to $\left\{C_{t}\right\}_{t \in T}$ is the family of Jacobian varieties

$$
g=\bigcup_{t \in T} J\left(C_{t}\right)
$$

where care must be taken to insert the generalized Jacobian of $C_{t_{\alpha}}$ over the critical points. When this is done we obtain a complex manifold $\mathscr{f}$ that is a fibre space

$$
\pi: \mathscr{g} \rightarrow T
$$

of commutative complex Lie groups.

Definition. A normal function $\nu$ is a holomorphic cross-section

$$
t \rightarrow \nu(t) \in J\left(C_{t}\right)
$$

of the fibre space (16). 
To see how such a normal function might arise we consider an algebraic curve $D$ on $S$ that meets each plane section (15) in points, and we write the intersection as

where

$$
D \cdot C_{t}=\sum_{i} p_{i}(t)
$$

$$
p_{i}(t)=\left(x_{i}(t), y_{i}(t), t\right) \text {. }
$$

We assume chosen a rationally varying set

$$
\omega_{\alpha}(t)=\frac{q_{\alpha}(x, y, t) d x}{p_{y}(x, y, t)}, \quad \alpha=1, \ldots, g=\text { genus } C_{t},
$$

of abelian differentials that are holomorphic and linearly independent on a general $C_{t}$, and then the curve $D$ defines a normal function $\nu_{D}$ by

$$
\nu_{D}(t)=\left(\sum_{i} \int_{p_{0}}^{p_{i}(t)} \omega_{1}(t), \ldots, \sum_{i} \int_{p_{0}}^{p_{i}(t)} \omega_{g}(t)\right)
$$

where $p_{0}$ is a base point of the pencil. Intuitively speaking, normal functions represent an extension to curves varying algebraically on parameters of the theory of abelian integrals on a fixed curve.

In this vein, a fundamental result is the following extension of the Jacobi inversion theorem (4):

(18) PoINCARÉ's EXISTENCE THEOREM. In case the fibre space (16) arises from a general pencil of curves on a surface, any normal function $\nu$ arises from a global algebraic curve $D$ on the surface; i.e., $\nu=\nu_{D}$.

As we shall see below (cf. (19)), normal functions are essentially linear data (analogous to sections of vector bundles), and the conversion to a geometric object provided by (18) is indeed quite remarkable.

For an application, we recall that the irregularity $q$ of an algebraic surface is equal to number of linearly independent closed holomorphic 1-forms on the surface. Keep in mind also that the development being described occurred some 25 years before Hodge theory and the equality $h^{0}\left(\Omega_{S}^{1}\right)=h^{1}\left(O_{S}\right)$. Using (18) Poincaré gave the first complete proof of the existence of $\infty^{q}$ algebraically equivalent but linearly inequivalent curves on the surface, as follows: Using the complete reducibility theorem (1) we choose the $\omega_{\alpha}(t)$ to be divided into two sets

$$
\underbrace{\omega_{1}(t), \ldots, \omega_{q}(t)}_{\text {fixed part }} ; \quad \underbrace{\omega_{q+1}(t), \ldots, \omega_{g}(t)}_{\text {variable part }}
$$

where the first set consists of the restrictions to $C_{t}$ of the holomorphic 1-forms on $S$ (Picard proved that this restriction is injective), and where the remainder is a complementary set as furnished by (1). The periods of the fixed part are constant, and from this it follows that for any algebraic curve $D$ on $S$ the first $q$ abelian sums

$$
\sum_{i} \int_{p_{0}}^{p_{i}(t)} \omega_{1}(t)=c_{1}, \ldots, \sum_{i} \int_{p_{0}}^{p_{i}(t)} \omega_{q}(t)=c_{q}
$$


give a constant vector $c=\left(c_{1}, \ldots, c_{q}\right)$. Moreover, we may vary the normal function by varying the constant vector $c$ while leaving fixed the entries of $\nu_{D}(t)$ corresponding to the variable part. Appealing now to (18), Poincaré established the existence of $\infty^{q}$ curves $D(c)$ that are easily seen to be linearly inequivalent.

(e) To illustrate the fertility of normal functions, we shall very briefly recount some of the developments that have occurred since Poincarés papers and shall mention a couple of rather general and seemingly difficult outstanding problems.

The first and foremost development is the Lefschetz $(1,1)$ theorem [12]. As above, on a smooth surface $S$ we consider a general pencil $\left\{C_{t}\right\}$ of hyperplane sections. The following was proved by Lefschetz (cf. [11 and 13] for expository accounts):

(19) To each normal function $\nu$ these is associated a topological invariant, called its fundamental class

$$
\eta(\nu) \in H^{2}(S, \mathbf{Z})
$$

that has the following properties:

(i) In case $\nu=\nu_{D}$ comes from an algebraic curve $D$ on the surface,

$$
\eta\left(\nu_{D}\right)=\eta(D)
$$

is the fundamental class of the curve; and

(ii) a given class $\eta \in H^{2}(S, \mathbf{Z})$ is the fundamental class $\eta(\nu)$ for some normal function $\nu$ if, and only if, $\eta$ has Hodge type $(1,1)$.

Denoting by $\operatorname{Pic}^{0}(S)$ the $q$-dimensional Picard variety of $S$, the situation may be summarized by the following exact sequence:

$$
\begin{array}{r}
0 \rightarrow \operatorname{Pic}^{0}(S) \rightarrow\{\text { group of normal functions }\} \\
\stackrel{\eta}{\rightarrow} H_{\text {prim }}^{2}(S, \mathbf{Z}) \rightarrow H^{0,2}(S)
\end{array}
$$

where $H_{\text {prim }}^{2}(S, \mathbf{Z})$ is the primitive part of $H^{2}(S, \mathbf{Z})$.

The Lefschetz $(1,1)$ theorem, which characterizes the fundamental classes of algebraic 1-cycles on $S$ by their Hodge types, is an immediate corollary of (20) together with Poincaré's existence theorem.

The remaining developments that we shall describe deal with higher-dimensional varieties. To a smooth complex algebraic variety $V$ we associate the following groups:

$$
\begin{aligned}
& Z^{n}=\{\text { algebraic cycles of codimension } n \text { on } V\}, \\
& \cup \\
& Z_{h}^{n}=\{\text { cycles homologous to zero }\} \\
& \cup \\
& Z_{a}^{n}=\{\text { cycles algebraically equivalent to zero }\}, \\
& \cup \\
& Z_{r}^{n}=\{\text { cycles rationally equivalent to zero }\} .
\end{aligned}
$$

The $n$th intermediate Jacobian $J^{n}(V)$ consists of the real torus

$$
H^{2 n-1}(V, \mathbf{R}) / H^{2 n-1}(V, \mathbf{Z})
$$


provided with the complex structure indicated by the following splitting of $H^{2 n-1}(V, \mathbf{R}) \otimes \mathbf{C} \cong H^{2 n-1}(V, \mathbf{C})$ into complex conjugate subspaces:

$$
\left\{\begin{array}{l}
H^{2 n-1}(V, \mathbf{C})=H^{\prime}(V) \oplus H^{\prime \prime}(V), \\
H^{\prime}(V)=H^{2 n-1,0}(V) \oplus \cdots \oplus H^{n, n-1}(V), \\
H^{\prime \prime}(V)=\overline{H^{\prime}}(V)
\end{array}\right.
$$

Choosing a basis $\omega_{1}, \ldots, \omega_{g}$ for $H^{\prime \prime}(V)^{*}$, there is a mapping

$$
u: Z_{h}^{n} \rightarrow J^{n}(V)
$$

given by

$$
u(z)=\left(\int_{\Gamma} \omega_{1}, \ldots, \int_{\Gamma} \omega_{g}\right)
$$

where $z \in Z^{n}$ is an algebraic $n$-cycle that is homologous to zero and $\Gamma$ is a chain with $\partial \Gamma=z$. For divisors on curves, (21) reduces to the usual abelian sums. An easy generalization of one-half of Abel's theorem implies that

$$
u\left(Z_{r}^{n}\right)=0
$$

so that there is an induced mapping

$$
u: Z_{h}^{n} / Z_{r}^{n} \rightarrow J^{n}(V) .
$$

For simplicity of notation we shall henceforth concentrate on the crucial case when

$$
\operatorname{dim} V=2 m-1, \quad n=m,
$$

and we shall drop the superscript $n$ (for example, one may think of algebraic curves on a threefold). As was the case for curves, one may speak of algebraic families $\left\{V_{t}\right\}_{t \in T}$ whose general member is smooth, and also (with some care) of the associated family $\left\{J\left(V_{t}\right)\right\}_{t \in T}$ of intermediate Jacobians. Provided that the singularities of the $V_{t_{\alpha}}$ are not too bad it can be shown that, again as was the case for curves, we arrive at a holomorphic fibre space

$$
\pi: \mathscr{G} \rightarrow T
$$

of commutative complex Lie groups with

$$
\pi^{-1}(t)=J\left(V_{t}\right)
$$

for $t \neq t_{1}, \ldots, t_{N}$ a noncritical parameter point.

Suppose now that $X$ is a smooth $2 m$-dimensional variety and $\left\{V_{t}\right\}$ is a general pencil of hyperplane sections. An algebraic $m$-cycle $D$ on $X$ is said to be primitive in case

$$
D \cdot V_{t}=Z_{t}
$$

is homologous to zero on $V_{t}$. There is then a corresponding normal function $\nu_{D}$ defined by

$$
\nu_{D}(t)=u_{t}\left(z_{t}\right) \in J\left(V_{t}\right)
$$

As is perhaps suggested by the formal nature of the exact sequence (20), the Lefschetz theorem (19) goes over pretty much verbatim, and we shall give in (23) below that part of the generalization of (20) that is relevant to our 
purposes, referring to [14 and 15] for precise statements and details of the proof

$$
\left\{\begin{array}{l}
\text { group of } \\
\text { normal functions }
\end{array}\right\} \rightarrow H_{\text {prim }}^{2 m}(X, \mathbf{Z}) \rightarrow \underbrace{H^{m-1, m+1}(X) \oplus \cdots \oplus H^{0,2 m}(X)} \text {. }
$$

A corollary of (23) is

(24) $A$ class $\eta \in H_{\text {prim }}^{2 m}(X, Z)$ is the fundamental class of a normal function if, and only if, $\eta$ has Hodge type $(m, m)$.

In particular, what is missing for the construction of algebraic cycles is a generalization of Poincaré's existence theorem (18).

Now Poincaré's existence theorem was given as a version of the Jacobi inversion theorem with dependence on parameters, but the latter entirely changes character in higher dimensions. For example, in [8] it is proved that

(25) If $V$ is a generic member of a sufficiently ample pencil $\left\{V_{t}\right\}$ on $X$, and if $m \geqslant 2$, then the mapping

$$
u: Z_{a} / Z_{r} \rightarrow J(V)
$$

is zero.

A consequence is that the subgroup of invertible points in $J(V)$, defined to be the image of the mapping (22), will be countable. That it is not always zero may be seen using (24) (cf. [8]).

In general we shall use the notations

$$
\begin{gathered}
J_{0}(V)=\text { image of the mapping }(22) \\
=\left\{\begin{array}{l}
\text { "continuous part" of the } \\
\text { invertible subgroup of } J(V) ; \\
\Theta(V)=Z_{h} / Z_{a} .
\end{array}\right.
\end{gathered}
$$

(27) Problem. (i) Is the group $\Theta(V)$ finitely generated?

(ii) Is the induced mapping

$$
u: \Theta(V) \rightarrow J(V) / J_{0}(V)
$$

injective?

If (ii) were true, then (i) would be a Mordell-Weil type question and would consequently seem to be more tractable. As it stands the above problem is probably too difficult, and so it might be profitable to try some special cases, such as a smooth quintic hypersurface in $\mathbf{P}^{4}$ or one of Clemens' double solids [16]. We remark that a normal function analogue of (27) is true (cf. [17]).

Having commented on the apparent demise, in higher dimensions, of the Jacobi inversion theorem for sufficiently general varieties (however, cf. [18]), it may still be asked if the analogue of Poincaré's existence theorem could be established by some other method? According to (24), the assumption that an integral cohomology class has Hodge type $(m, m)$ has at least led to the construction of a global analytic variety somewhere, namely the graph of the normal function $\nu$. Moreover, in case $\nu=\nu_{D}$ for some primitive algebraic $m$-cycle $D$ it may be proved that the knowledge of $\nu$ in a sense determines the 
hypersurfaces of sufficiently high degree that pass through $D$ or a cycle homologous to it [13]. Unfortunately, as discussed at the end of [13] this construction does not seem likely to be of much help in constructing cycles. Perhaps more promising is the possibility of relating the infinitesimal properties of $\nu_{D}$ to the geometry of $D$, and we shall briefly describe this recent development.

As initial motivation, suppose that $X \subset \mathbf{P}^{N}$ is a smooth algebraic surface and $D_{0} \subset X$ is a divisor of degree zero (e.g., the difference of lines from the two rulings of a quadric surface in $\mathbf{P}^{3}$ ). Write $D_{0}=D_{0}^{+}-D_{0}^{-}$as the difference of effective divisors and denote by $\sigma\left(D_{0}\right)=D_{0}^{+}+D_{0}^{-}$the support of $D_{0}$. If $C \in\left|\vartheta_{X}(d)\right|$ is a general section of $X$ by a hypersurface of degree $d$, then for $d \gg 0$ the divisors $D_{0}^{ \pm} \cdot C$ are effective special divisors, and the variational theory of special linear systems (cf. Chapter 7 of [20]) shows that there are a priori conditions on the tangent spaces to the graph of $\nu_{D_{0}}$. Moreover, in a very few cases these infinitesimal conditions may be used to single out the adjoint hypersurfaces of large degree that pass through one or more of the curves $\sigma(D)$ where $D=D^{+}-D^{\prime}$ is homologous to $D_{0}$ and $\operatorname{deg} D^{+}=\operatorname{deg} D_{0}^{+}$(cf. [19]).

As another motivation, suppose that $X \subset \mathbf{P}^{5}$ is a smooth hypersurface of degree $d$ that contains a 2-plane $\Lambda \cong \mathbf{P}^{2}$. Then

$$
D=d \cdot \Lambda-\left(\mathbf{P}^{3} \cdot X\right)
$$

will be a primitive algebraic 2-cycle lying in $X$, and we may consider the normal function corresponding to the linear sections

$$
V_{H}=H \cap X, \quad H \in \mathbf{P}^{5^{*}},
$$

of $X$. In (c) above we discussed how the holomorphic 1-forms on $J\left(V_{H}\right)$ can be represented, via the isomorphism

$$
H^{1,0}\left(J\left(V_{H}\right)\right) \cong H^{\prime}\left(V_{H}\right)
$$

by residues of the form

$$
\operatorname{Res}\left(\frac{q(x) d x_{1} \wedge d x_{2} \wedge d x_{3} \wedge d x_{4}}{p(x)^{2}}\right), \quad \operatorname{deg} q(x) \leqslant 2 d-5,
$$

where $p(x)=0$ is the defining equation of $V_{H} \cap H$. It is certainly plausible that the derivative of the individual integrals in (21) should "change character" where the polynomial $q(x)$ vanishes on the line $\Lambda \cap V_{H}$.

We shall now sketch the definition of an infinitesimal invariant $\delta \nu$ of a normal function $\nu$ and shall then give one illustrative result. Let $\left\{V_{s}\right\}_{s \in S}$ be a family of smooth varieties of dimension $2 m-1$ and $\nu(s) \in J\left(V_{s}\right)\left(=J^{m}\left(V_{s}\right)\right)$ a normal function. Even in the case $m=1$ of curves of genus $g$, the construction of $\delta \nu$ is somewhat complicated due to the fact that, even though the Siegel generalized upper-half-plane $\mathcal{H}_{g}$ is a homogeneous complex manifold, the versal family of principally polarized abelian varieties over $\mathcal{H}_{g}$ is not (otherwise, all theta divisors would look alike). Because the construction is 
local we may assume that $S \subset \mathbf{C}^{N}$ is a polydisc and we may then naturally identify all the groups $H^{2 m-1}\left(V_{s}, \mathbf{Z}\right) /\left(\right.$ torsion) with a fixed lattice $\mathbf{Z}^{2 g}$ having complexification

$$
\mathrm{C}^{2 g} \cong H^{2 m-1}\left(V_{s}, \mathrm{C}\right)=H^{\prime}\left(V_{s}\right) \oplus H^{\prime \prime}\left(V_{s}\right) .
$$

Then $H^{\prime}\left(V_{s}\right) \subset H^{2 m-1}\left(V_{s}, \mathrm{C}\right)$ gives a holomorphically varying $g$-plane

$$
\Lambda_{s} \subset \mathbf{C}^{2 g} \text {, }
$$

and we may consider the resulting map

$$
\varphi: S \rightarrow G(g, 2 g)
$$

given by $\varphi(s)=\Lambda_{s}$ (when $m=1, \varphi(s)$ is just the period matrix of $V_{s}$ under the natural embedding $\mathcal{H}_{g} \subset G(g, 2 g)$ ). Since $J\left(V_{s}\right)$ is the middle-dimensional intermediate Jacobian there is a natural identification

$$
H^{2 m-1}(V, \mathrm{C}) / H^{\prime}\left(V_{s}\right) \cong H^{\prime}\left(V_{s}\right)^{*},
$$

and so the differential of $(28)$ is given by

$$
\varphi_{*}: T_{s}(S) \rightarrow \operatorname{Hom}\left(\Lambda_{s}, \Lambda_{s}^{*}\right) .
$$

We define

$$
\Xi_{s} \subset \mathbf{P}\left(T_{s}(S)\right)
$$

by the condition

$$
\xi \in \Xi_{s} \Leftrightarrow \operatorname{det} \varphi_{*}(\xi)=0
$$

where $\xi \in \mathbf{P}\left(T_{s}(S)\right)$ is a tangent direction. Setting

$$
\Xi=\bigcup_{s \in S} \Xi_{s}
$$

the quotient spaces

$$
\mathrm{C}^{2 g} / \operatorname{span}\left\{\Lambda_{s}, \varphi_{*}(\xi) \Lambda_{s}\right\}
$$

fit together to define a coherent sheaf $\mathscr{F}$ over the analytic variety $\Xi$. The normal function is defined by giving

$$
\nu(s) \in \mathbf{Z}^{2 g} \backslash \mathbf{C}^{2 g} / \Lambda_{s}
$$

for each $s \in S$, and we choose an arbitrary lifting $v(s) \in \mathbf{C}^{2 g}$ of $\nu(s)$. Any other lifting is

$$
\tilde{v}(s)=v(s)+\lambda(s)+\zeta(s)
$$

where $\lambda(s) \in \Lambda_{s}$ and $\zeta(s) \in \mathbf{Z}^{2 g}$. It follows that, at the point $(s, \xi) \in \mathbf{P}\left(T_{s}(S)\right)$, the projection of $d v(s) / d \xi$ into the quotient space (30) depends only on $v$, up to a scalar resulting from lifting $\xi$ to a tangent vector in $T_{s}(S)$. We define the infinitesimal invariant

$$
\delta v \in H^{0}(\Xi, \mathscr{F}(1))
$$

to be the resulting section of $\mathscr{F} \otimes \vartheta(1)$, where $\theta(1)$ is the tautological line bundle over $\mathbf{P}(T(S))$. The details and differential geometric motivation for 
this construction may be found in [19], where also a proof of the following result may be found.

Let $C$ be a smooth curve and $D=D^{+}+D^{-}$a fixed divisor of degree zero with support $\sigma(D)=D^{+}+D^{-}$. For our family $\left\{C_{s}\right\}_{s \in S}$ we take the Kuranishi space (local moduli space) centered at $C_{s_{0}}=C$. Then there is a natural identification

$$
T_{s_{0}}(S) \cong H^{1}(C, \Theta)
$$

where $\Theta$ is the tangent sheaf of $C$. We note that

$$
\mathbf{P} H^{1}(C, \Theta) \cong \mathbf{P}^{3 g-4}
$$

is the embedding space for the bicanonical mapping

$$
\varphi_{2 K}: C \rightarrow \mathbf{P} H^{1}(C, \Theta)
$$

and it may be proved that (cf. [19])

$$
\Xi_{s_{0}}=\bigcup_{E \in|K|} \overline{\varphi_{2 K}(E)}
$$

where $|K|$ is the canonical linear series on $C$ and $\overline{\varphi_{2 K}(E)}$ is the linear span of the points $\varphi_{2 K}\left(p_{i}\right)$ where $E=p_{1}+\cdots+p_{2 g-2}$. We remark that if $\xi \in$ $\mathbf{P}\left(T_{s_{0}}(S)\right)$ is in $\varphi_{2 K}(E)$, then

$$
H^{0}(C, K(-E)) \subset \operatorname{ker} \varphi_{*}(\xi) \text {. }
$$

Moreover, given $\omega \in H^{0}(C, K(-E))$ and any vector $f$ in the quotient space (30), the pairing ( $Q$ denoting the cup-product on $H^{1}(C)$ )

$$
Q(\omega, f)
$$

is well defined since $\varphi_{*}(\xi)$ is a symmetric transformation, and consequently

$$
\operatorname{span}\left\{\Lambda_{s^{\prime}}, \varphi_{*}(\xi) \Lambda_{s_{0}}\right\}=\Lambda_{s_{0}}^{\perp} .
$$

Suppose now we consider families of divisors $D_{s}$ on $C_{s}$ with $D_{s_{0}}=D$ and denote by $\nu_{D_{s}}$ the resulting normal function. By what we just said, to know the value of the infinitesimal invariant (31) at $s=s_{0}$ it will suffice to know the quantities

$$
Q\left(\omega, \delta \nu_{D_{s}}\right) \text { at } s=s_{0},
$$

where $\xi \in \overline{\varphi_{2 K}(E)}$ and $\omega \in H^{0}(C, K(-E))$. These quantities are evaluated in [19], and in particular the conditions that

$$
Q\left(\omega, \delta \nu_{D_{s}}\right)=0 \text { at } s=s_{0}
$$

are determined. Here we mention the two conclusions:

(i) the geometric condition

$$
(\omega) \geqslant \sigma(D)
$$

implies (33), and

(ii) if $D_{s}$ is chosen arbitrarily subject to $D_{s}=D$, then (33) and (34) are equivalent. Thus, in a certain sense $\delta \nu$ gives an extension to a family of variable curves of the Brill-Noether matrix (cf. [20]) that plays the pivotal role in the theory of special divisors on a fixed curve. 
Although somewhat complicated to describe, we have mentioned this result as perhaps indicating that Poincarés concept of normal functions has signficance that may play a role in questions of current interest in algebraic geometry.

\section{REFERENCES}

(a) Some sample works of the period.

1. P. Appell and E. Goursat, Théorie des fonctions algébriques et de leurs intégrales, GauthierVillars, Paris, 1929.

2. G. Castelnuovo and F. Enriques, Grundeigeshaften der Algebraischen Flächen, Encyklop. der Math. Wissenschaften, 3 (1903), 635-768.

3. E. Picard and G. Simart, Theorie des fonctions algébriques de deux variables independentes, Gauthier-Villars, Paris, 1897-1906.

(b) References on abelian varieties.

4. P. Griffiths and J. Harris, Principles of algebraic geometry, Wiley, 1978 (cf. Chapter 2; our notations concerning curves and Jacobians are the same as this reference).

5. H. Poincaré, Sur les fonctions abéliennes, Amer. J. Math. 8 (1886), 289-342.

6. __ Remarques diverses sur les fonctions abéliennes, J. de Math., $5_{-}^{\mathrm{e}}$ series, 1 (1895), $219-314$.

(c) References on residues.

7. H. Poincaré, Sur les résidues des intégrales doubles, Acta Math. 9 (1887), 321-380.

8. P. Griffiths, On the periods of certain rational integrals. I, II, Ann. of Math. (2) 90 (1969), 460-495; 496-541.

(d) References on normal functions.

9. H. Poincaré, Sur les courbes tracées sur les surfaces algébriques, Ann. Sci. de l'École Norm. Sup. 27 (1910), 55-108.

10. Sur les courbes tracées sur une surface algébrique, Sitz. der Berliner Math. Gesellschaft. 10 (1911), 28-55.

(e) References on recent developments.

11. O. Zariski, Algebraic surfaces, Springer-Verlag, Berlin and New York, 1971 (cf. especially Chapter VII).

12. S. Lefschetz, l'Analysis situs et la géometrié algébrique, Gauthier-Villars, Paris, 1924.

13. P. Griffiths, $A$ theorem concerning the differential equations satisfied by normal functions associated to algebraic cycles, Amer. J. Math. 101 (1979), 94-131.

14. S. Zucker, Generalized intermediate Jacobians and the theorem on normal functions, Invent. Math. 33 (1976), 185-222.

15. __ Hodge theory with degenerating coefficients: $L_{2}$ cohomology in the Poincare metric, Ann. of Math. (2) 109 (1979), 415-476.

16. C. H. Clemens, Double solids, Adv. in Math. (to appear).

17. P. Griffiths, Periods of integrals on algebraic manifolds. III, Publ. Math. Inst. Hautes Études Sci. 38 (1970), 125-180.

18. S. Zucker, The Hodge conjecture for cubic fourfolds, Compositio Math. 34 (1977), 199-209.

19. P. Griffiths, An infinitesimal invariant of normal functions (to appear).

20. E. Arbarello, M. Cornalba, P. Griffiths, and J. Harris, Topics in algebraic curves, Princeton Math. Series, Princeton Univ. Press, Princeton, N. J. (to appear)

Department of Mathematics, Harvard University, Cambridge, Massachusetts 02138 
